\title{
Modeling an Agile Enterprise: Reconciling Systems and Process Thinking
}

\author{
Ilia Bider ${ }^{1,2}$, Gene Bellinger $^{3}$, and Erik Perjons ${ }^{1}$ \\ ${ }^{1}$ DSV, Stockholm University, Stockholm, Forum 100, SE-16440 Kista, Sweden \\ ${ }^{2}$ IbisSoft AB, Stockholm, Box 19567, SE-10432 Stockholm, Sweden \\ ${ }^{3}$ SystemsWiki.org \\ ilia@ibissoft.se, systemswiki@gmail.com, perjons@dsv.su.se
}

\begin{abstract}
Selection of an enterprise modeling paradigm depends on the practical task the modeling project is trying to achieve. For example, modeling in the frame of Enterprise Architecture paradigm is, usually, aimed at alignment of the enterprise components, such as mission, vision, business processes, services and IT systems. Modeling in the frame of Business Process Management is aimed at process improvement/optimization, and modeling in the Systems Thinking paradigm is aimed at getting a holistic view on the dynamic behavior of the enterprise. This paper suggests a new approach to enterprise modeling that combine these approaches in order to reveal and improve enterprise agility. It is based on the systemic view of business processes, and it presents an enterprise as a three-layered model consisting of assets, sensors and business process instances. Elements of this model can be recursively decomposed, which allows for different levels of details when modeling an enterprise.
\end{abstract}

Keywords: Business Process Management, Systems Thinking, Enterprise Agility.

\section{Introduction}

We start with two sayings that concern modeling. The first one runs as "All models are wrong, but some are useful" and is attributed to George E.P. Box [1]. The second one runs as "A method that is good for everything is good for nothing". The meaning of these two sayings in the context of the PoEM conference can be expressed as each enterprise modeling technique can be useful to solve some practical problems in certain context, but might be useless, or even counterproductive for solving other problems, or problems in another context.

Consider the three paradigms most frequently used for modeling enterprises: Business Process Management [2], [3], Enterprise Architecture [4], [5], and Systems Thinking [6], [7], each of them having countless techniques and methods. Each of these paradigms is connected to a special problem area. Business Process Management is associated with optimization of the usage of resources inside an enterprise through specialization, standardization, and automation [8]. Enterprise 
Architecture is associated with alignment of different parts of the enterprise, like mission, vision, structure, processes and IT systems [5]. Systems Thinking is associated with a holistic view on the dynamic behavior of the enterprise and its interaction with its environment [6]. It is directed to avoiding situations in which the dynamic behaviors existing in different parts of the enterprise, e.g., sales and production, are disconnected. For example, sales is increasing its efforts, while production is pursuing cost savings by diminishing current production levels, which after some time, results in increasing time from order to delivery.

The scope in which the above paradigms are applicable may also differ. For example, a Business Process Management project can have an enterprise-wide scope and be aimed at identifying and modeling all processes in the organization. On the other hand, the project can concern a particular process, e.g., sales, in order to optimize it. The same is true for Systems Thinking. The project can be started to overhaul the whole enterprise, or just to understand and improve a particular situation. Enterprise Architecture paradigm differs from the previous two in this aspect, it is aimed to align all components of the organization and thus, usually require a large scale project that concerns the whole enterprise To complete an Enterprise Architecture project requires time and considerable resources which leads to this paradigm normally being used by large enterprises in sectors considered to be stable, energy, large industrial enterprises, financial sector, large governmental organizations.

None of the above paradigms focuses specifically on the issue of enterprise/business agility - property of an enterprise to function in the highly dynamic world [9]. The agility concerns both being able to adjust the enterprise to changes in the surrounding environment, and discovering new opportunities constantly appearing in the dynamic world for launching completely new products/services. Becoming agile requires a structure that allows discovering changes and opportunities as soon as possible and react on them appropriately. A model that reveals the current level of agility, and can show ways of improving it would be beneficial for a new generation of highly dynamic team based enterprises.

This paper is aimed at presenting an idea of how a enterprise model explicitly related to issues of enterprise agility could look like. The model can be the base for a new modeling technique managing agility. While creating this model, we do not reject the concepts and features existing in the other three paradigms. Actually, we borrow all we can and adjust and integrate the borrowed concepts in a manner that is appropriate for the goal our model is aimed to serve. For example, a reaction on a discovered change is defined as starting a business process instance/case. However, we do not insist that this process should be optimal, or need to be optimized in the future. It is enough if the process is very loosely defined so that an instance of such a process can be completed in a relatively short time. Optimization may not be appropriate in the dynamic environment as it will hinder the creativity needed for handling a particular process instance.

This work has its roots in the heated discussions in the LinkedIn group Systems Thinking World [10] on whether terms system and process represent the same or different concepts. These discussions resulted in our creation of the model managing enterprise agility. The model is based on applying a systemic view to business 
processes. Standard for Enterprise Architecture concepts as mission, vision, policies, structures, are taken into consideration, but are not set in the focus. The resulting enterprise model consists of three layers:

- Enterprise assets

- Sensors

- Business process instances

The rest of the paper is structured according to the following plan. In Section 2, we present a systemic perspective on business processes. Based on this perspective, in Section 3, we introduce the three-layered model for managing enterprise agility as mentioned above. In Section 4, we introduced a simplified classification of business processes based on the nature of their goals. In section 5, we explain how elements of different layers of the model interact with each other. In section 6, we discuss how sensors and business process instances can be recursively decomposed. In section 7 , we present some informal examples of application of the ideas introduced in the previous sections. Section 8 is devoted to related work. In section 9, we discuss the implications of the three-layered model, and draw plans for the future that concern the transition from the idea presented in the paper to the practical methodology of enterprise analysis and modeling.

\section{Systemic View on Business Processes}

According to Systems Thinking [6,7] an enterprise is regarded as a whole, i.e., a system. Such a system maintains its existence through constant interaction between its parts, i.e., people, departments, teams, etc. The system interacts also with its environment, a bigger whole, manifesting a unique behavior that cannot be derived from the sum of its parts. From a Business Process Management perspective [3], an enterprise is regarded as a number of repeatable business processes. These two perspective looks on the surface completely different, and to integrate both of them in a new modeling technique we need, first, to reconcile them. The reconciliation is done via considering processes as a special type of systems.

The term business process encompasses two concepts (which often confuse outsiders):

- business process instance (BPI) or case, for example, delivering a service to a particular customer after receiving a call for service

- business process type (BPT) or template which refers to all possible instances of a particular kind, e.g., all service delivery instances.

A business process instance (BPI) is a system with a short lifespan. It can be minutes, hours, days, months or years, but its lifespan is always (considerably) shorter than the lifetime of the whole enterprise. Such a system is created to achieve some (operational) goal, e.g., to deliver service, or goods ordered by a given customer and get paid. This system is disbanded after the goal has been reached. A BPI system includes the same components as the whole enterprise system (e.g., people, 
departments) and some elements of the environment (e.g., customers, investors). At any moment of time, an enterprise has numerous BPIs in progress providing the majority of the interactions between the system's components and the system, and the environment.

A business process type (BPT) can be considered as a set of rules that describes the dynamic behavior of BPIs aimed at reaching a certain class of operational goals. A BPT consists of two parts:

- Start conditions that defines when a new BPI system of the given type should be created

- Execution rules that define what should be the goal of the process, whom should be included in the process, how the job should be done in the process, how components interact, etc.

The execution rules can be prescriptive (e.g., what should be done), constraint-based restrictive (e.g., what should never be done), recommended (e.g., how normally things are done but it is allowed to do it differently), or a combination of the above. BPT rules are "imprinted" in manuals, process maps, employees' handbooks, computer systems, heads of employees (oral tradition), or a combination of the above. In other words, the knowledge on the rules (and the processes themselves) can range from being completely tacit (e.g., resides in the heads of the process participants), to partly or totally explicit (e.g., depicted in detailed process maps).

BPTs work as business DNA creating BPIs based on the needs, e.g., impulses or changes in the environment or inside the organizational system itself. BPTs plus tools used in BPIs, e.g., telephone lines, computers, production lines, constitute an organizational system's infrastructure that allows it to effectively function inside the given environment. BPTs constitute some kind of hierarchy. The lower levels are occupied by BPTs that produce BPIs as a reaction to simple impulses, like incoming order from a customer. The higher levels are occupied by more strategic BPTs that react on more substantial changes in the environment by reconfiguring the system itself, which may include changing BPTs (a kind of genetic engineering), introducing new BPTs, or deleting the obsolete ones.

\section{The Three-Layered Enterprise Model}

Based on the elaboration in Section 2, we can view an organization as a three-layered model as depicted in Fig. 1, and explained below:

1. An assets layer consists of:

- People - with their knowledge and practical experiences, beliefs, culture, sets of values, etc.

- Physical artifacts - such as computers, telephone lines, production lines, etc.

- Organizational artifacts, formal as well as informal - such as departments, teams, networks, roles, etc. 
- Information artifacts - such as policy documents, manuals, process descriptions (BPTs), etc. To the information artifacts belong both written (documented) artifacts, and tacit artifacts - the ones that are imprinted in people's heads (e.g., culture.)

The assets layer is relatively static, which means that this layer by itself cannot change anything. The components of this layer are activated when they are included in the other two layers. This layer itself can be changed through other layers when the assets are set in motion for achieving some useful goals. Note that assets here are not regarded in pure mechanical terms. All "soft" assets, like sense of common goals, degree of collaboration, shared vision etc., belong to the organizational assets. Note also that having organizational artifacts does not imply a traditional function oriented structure. Any kind of informal network, or resource oriented structural unit, is considered as organizational artifact.

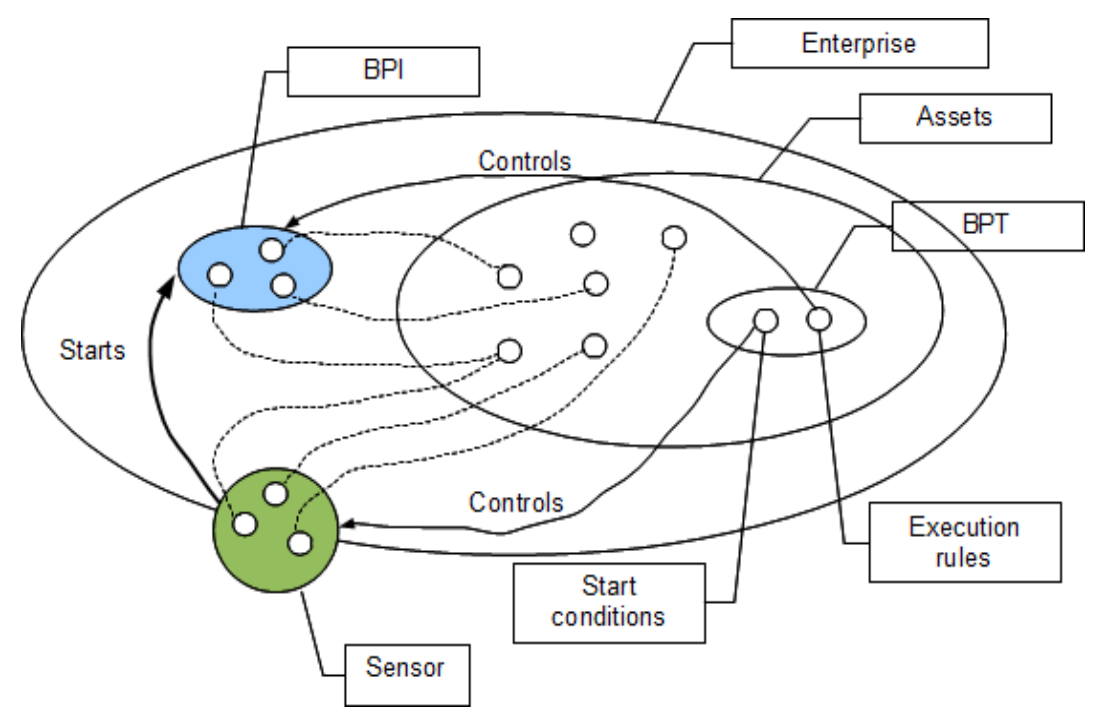

Fig. 1. Three-layered model of an enterprise as a system

2. Sensor layer consists of a set of (sub)systems, the goal of which is to watch the state of the enterprise itself and its environment in order to catch impulses and slower changes that require firing of business process instances of certain types. We need a sensor (which might be a distributed one) for each business process type. The work of a sensor is governed by the Start Conditions of the BPT description (which is an informational artifact). A sensor can be fully automatic for some processes (an order placed by a customer in a web-based shop), or require human participation to detect changes in the system or its surroundings.

3. BPIs layer - a set of systems initiated by sensors for reaching certain goals and disbanded when these goals are achieved. The behavior of a BPI system is governed by the Execution Rules of the corresponding BPT. Dependent on the type, BPIs can lead to changes being made in the assets layer. New people are 
hired or fired, departments are reorganized, roles are changed, new policies are adopted, BPT descriptions are changed, new BPTs are introduced, and obsolete ones are removed.

\section{Classification of Business Processes}

We can roughly differentiate three categories of business processes dependent on the complexity of the sensor, and the nature of the process itself:

1. The first category encompasses operational processes, like sales, production, HR (e.g., hiring), etc. A sensor discovers the need (e.g., customer needs - sales, or internal needs - HR), and initiates a relatively structured process instance to attain the operational goal (e.g., making a deal, or hiring a new employee).

2. The second category encompasses process improvement (optimization) processes. A sensor here is based on the performance indicators established to measure efficiency, productivity, or other parameters of the given BPT. If the performance is not according to the expectations, an improvement (re-engineering) BPI starts with the goal to change process definition(s) used by operational processes. The improvement BPI here can follow some known methods (like Six Sigma, or lean). As a rule, the improvement processes are less structured than the operational ones.

3. The third category encompasses strategic processes. A sensor here is based on the macro view on the whole organizations. If the overall performance is below expectation, a strategic BPI is fired with the goal of considerably changing the assets layer. This can include radical changes in process definitions, removing obsolete processes, introducing new ones, rearranging departments, substituting keymanagers, introducing new technology, etc. These are the processes where Systems Thinking is (though maybe too seldom) applied as guidelines for finding the best places to make changes (leverage points). A process here may be completely ad hoc, or use some loose structure, e.g., a series of brainstorming sessions.

\section{Interplay between the Layers}

Due to the interplay between the three layers, an enterprise behaves as an adaptive system. It constantly interacts with the environment based on the BPTs of operational processes, optimizes itself to the current environment through the improvement processes, and can reconfigure itself when the environment changes based on the strategic processes (after which it can start optimization to the new environment).

Basic interconnections between the elements of different layers are depicted in Fig. 1. These, and the additional ones that are not explicitly shown in Fig. 1, are explained below.

A BPI (layer 3) is started by the corresponding sensor (layer 2), and it uses organizational assets (layer 1), such as people, machines, etc. to produce the result, 
i.e., reach some operational goal. In particular, the execution rules of the BPT (layer 1) controls the behavior of each BPI that belongs to this process type.

As was already mentioned, a BPI can be started to intentionally change the organizational assets (layer 1), fire or hire people, change policies, change BPTs, reorganize departments, etc. But even when a BPI does none of the above, it does make a change just because it was running for some period of time. During its run, a BPI creates a trace either on paper, or inside the organizational database, or just in the heads of people participating in this process instance. Depending on the type of organizational memory, the trace may stay in memory a very short period of time, or last "forever". All remembered traces of BPIs constitute the experience based knowledge of the organization, which, as an information artifact, adds to the assets layer (layer 1).

A sensor (layer 2) starts a BPI (layer 3) each time it detects that its start conditions are satisfied, and it uses assets (layer 1), such as people, machines, etc., to detect this situation. In particular, the start conditions of the BPT (layer 1) controls the behavior of the sensor (see Fig. 1).

For a sensor to identify when a BPI is to be started, it needs access to the relevant information in order to test the start conditions. This information is usually delivered by some already finished BPIs (layer 3 ) and can be found in their traces (layer 1). The information that sensors need can be provided by normal BPIs aimed at practical goals (as their side effects). However, in many cases, just having standard operational business process is not enough to provide all information needed for the sensors. Special "information gathering" business processes need to be designed with the only goal of obtaining relevant information for the sensors. The start condition for such a business process could be very simple, a BPI should run each year, month, week, year, our hour. Information gathering processes are especially needed for the sensors belonging to the categories 2 and 3 of the process classification from Section 4. A periodical survey of the customers to determine their level of satisfaction is a typical example of an information gathering business processes.

Another way of obtaining information needed for sensors is to enhance the standard operational processes in order to gather this information during the BPIs runs. This can be done by adding to them steps (operations) that are not important for these processes as such, but can provide information for sensors belonging to other business processes. An example of such enhancement could be a set of questions to the potential customer who has chosen not to buy a product or service, just to find out the reason for his/her decision (wrong price range, wrong service, etc.).

\section{Decomposition}

Both a sensor and a BPI are systems, and thus they can be, if necessary, decomposed. Consider an example of a compound sensor. Let us have a fast growing enterprise that wants to keep the pace of its expansion for a number of years ahead. Let this enterprise be a consulting business, the growth of which depends on the number of employees. The management decides to run a strategic overview (BPI) according to some template (BPT) as soon as there is a danger for growth or decline (start condition of the BPT). One of the parameters that reflect the pace of growth is the rate 
of consultants hiring minus rate of losing them to competitors. A sensor needs to evaluate this data against some threshold value and start a strategic overview when the value is below the threshold. There could be two possibilities to create such a sensor:

- ensure that all needed information is available in real-time, then the sensor just needs to do some math and issue a start signal if necessary

- make a periodic, say once a month, information gathering, and only then do some math, and issue a start signal if necessary

The second case represents a compound sensor as in Fig. 2, which consists of a simple Sensor1 that "watches" the clock and starts an information gathering BPI on the 1st of each month. The BPI gathers information and produces a report (information artifact). Another sensor, Sensor2 in Fig. 2, reacts to a new report, makes comparisons and starts a new strategic overview if necessary.

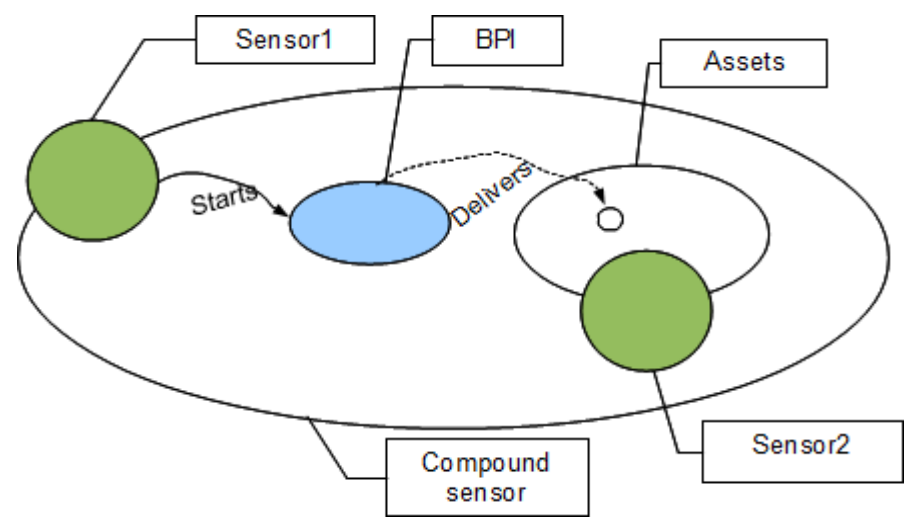

Fig. 2. A compound censor

A similar decomposition can be done for a BPI, for example, when the BPI represents a complex BPI, e.g., a project. Complex sensors/BPIs are more typical for the category 2 and 3 of the process classification in Section 4 than for the category 1 (operational processes).

\section{$7 \quad$ Applying the Model}

\section{1 "Analysis" of the Internet Bubble}

Consider a simplified, and a bit exaggerated, example of an IT consulting company with sales department, consulting department, and HR department. The company uses a "usual" business model of charging per hour based on the expert level of its consultants.

Sales department conducts sales process instances according to the sales BPT (definition), part of which is hourly pricing of consulting services according to the 
level of the consultants expertise. HR department is hiring consultants according to the hiring BPT (definition), part of which is salary ranges according to the experience and education of the consultants. Hiring is done to ensure growth or just compensate natural lost.

Suppose management discovers that HR hires fewer consultants than expected. An investigation shows that the reason is the company offering less competitive salaries than their competitors. The hiring BPT is revised and salary offers become higher. The rate of hiring returns to normal (expected).

Suppose that at the same time new sales started to decline. An investigation shows that the company chargers more per hour than their competitors. The sales BPT is revised and the company starts charging less per hour. The rate of getting new consulting assignment returns to normal (expected).

The above adjustment can go through several cycles until the "strategic sensor" catches a new trend: the revenues from new assignments do not cover costs for their completion. A strategic business process instance is fired to find the best leverage point(s) to solve the problem using some System Thinking technique. What would it be?

What happens if the strategic sensor discovers the above situation too late, or there is no strategic BPT at all, or the fired BPI is unsuccessful. Well, bad luck, the company goes out of business.

Does everything above sounds too simplified? It might be so, but in the late 1990s a lot of start-up Swedish IT consultancies operated in this manner until most of them went out of business when the IT-bubble burst. We are not stating here that the management of these companies did not know what they were doing, some of them knew. Their actions might have had a more rational behavior, like dumping prices in hope to get rid of the competitors while having enough of risk capital. This, however, did not matter much in the end.

The artificial example above shows that failing to have a proper sensor may result in a complete demise of an enterprise working in a highly dynamic environment. This example reveals the weakness of the traditional enterprise structure, in which each process type engages a separate set of people. Sales BPIs are manned with sales staff, hiring BPIs are manned with HR staff, process improvement BPIs are manned with the Process Office staff, strategic BPIs are manned with high-level management. An example of such structure is schematically shown in Fig. 3. This picture is less detailed than the one in Fig. 1. Here, a process circle encompasses both BPI and sensors.

There are two weaknesses in the traditional organization that are revealed by the example above:

- Parts of the systems are separated from each other and thus may easily drift apart destroying the system as was shown in the example in the previous section

- The traditionally built enterprise is vulnerable if it operates in a highly dynamic competitive environment. The whole structure will work fine provided that sensors discover emerging situations fast enough so that the organization have time to adjust. In the highly dynamic environment, the costs of creating such sensitive sensors might be too high to make the whole idea sustainable. 


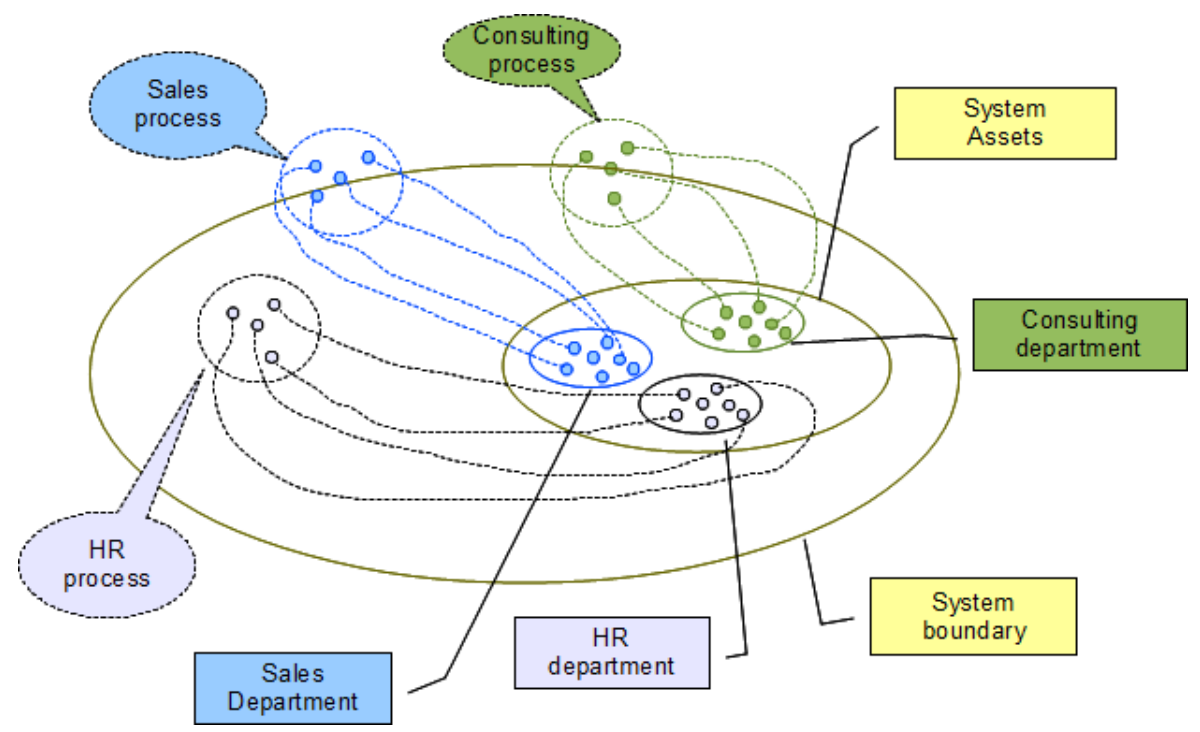

Fig. 3. Traditional enterprise structure from the process perspective

\subsection{Selling IT-consultants and Hire a Friend Principles}

As a reaction to what happened during the IT bubble (see section 9), the Swedish IT consulting industry adopted two new principles: "selling IT-consultants", and "hire a friend". On the conceptual level both principles mean removing the rule that different operational processes, e.g., sales, service delivery, hiring, are manned by different categories of people.

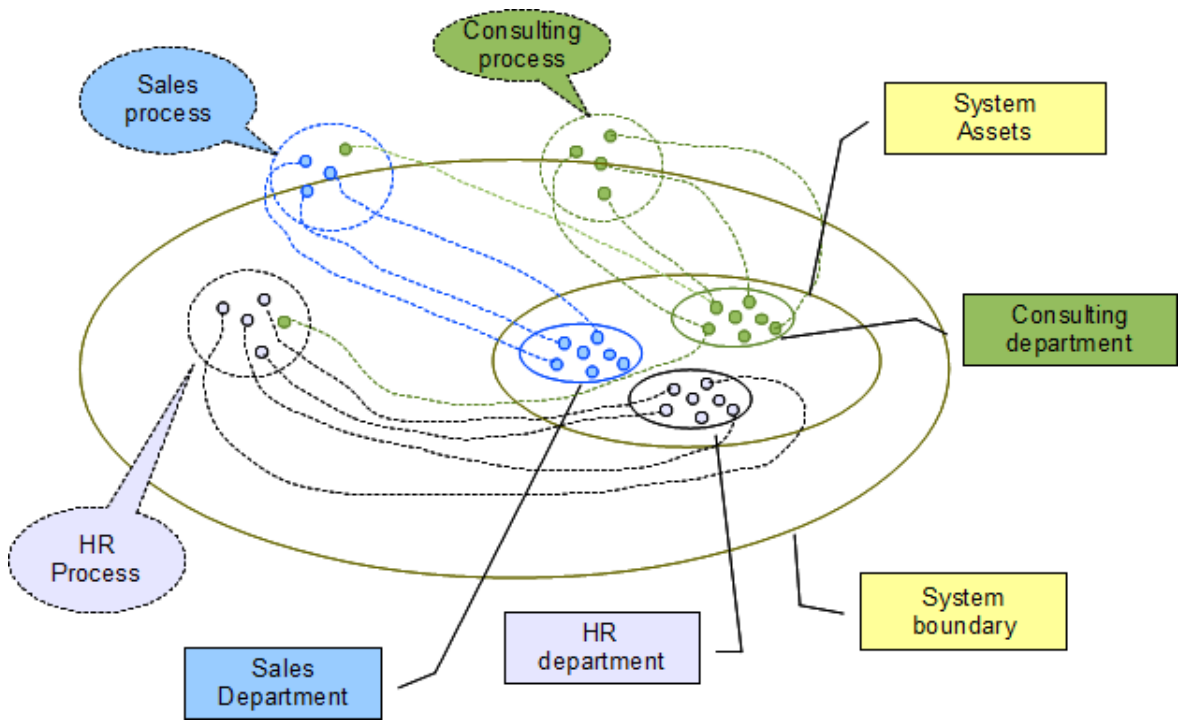

Fig. 4. Cross-manning of business processes 
It is not clear whether every IT consultant can be a good sales person, or a good HR person, but in the most cases, due to his/her positioning in the outer world, a consultant can greatly contribute to the sales and HR processes. To make use of his/her position, for example, in sales, the consultant's role in this process can include him/her serving as a part of the process "sensor" by creating leads:

- as a provider of information during the process, and

- as a promoter of the company's line of products and services.

The above does not mean that the sales-staff should disappear and all sales should be conducted by consultants. It means active engagement of other categories of professionals in the sales process on a regular (and not on an ad hoc) basis. Expected results are more sensitive, and less expensive sensors which will make the processes more efficient on the whole.

Selling consultants and hiring a friend represent examples of so-called crossmanning of business processes. A schematic representation of this concept is given in Fig. 4, which is modification of Fig. 3.

\subsection{Discussion}

An analysis of the examples above shows that the three-layer enterprise model can be applied on the conceptual level to discover and explain weaknesses and strong sides of different ways of organizing business. For example, it helps to understand and explain the essence of cross-manning. Cross-manning can be applied not only to the category 1 operational processes but also to category 2 - improvement processes. Instead of letting specially assigned process re-engineers make detailed process designs, why not let people engaged in these processes do, at least, part of the job themselves? Let process specialists and management devise basic guidelines, and let professionals on the floor fill in the details. The advantages are:

- people on the floor will know sooner when the old process definition stops to satisfy the internal or external environment (more sensitive sensor for the improvement process)

- as they know better not only the business, but also each other's capabilities, they are in a better position to adjust the definitions not only to the abstract goals but also to particular people that man the operational processes

\section{Related Research}

As was stated in the introduction, we derive our model from reconciliation of Systems and Process Thinking. Therefore, there are many features in our model that can be found elsewhere. We do not claim that all elements of our model are new; only a combination of them is to the best of our knowledge original. Not having enough space to review all related works, we will focus our attention only on two models that we consider most related to ours. One is the Viable Systems Model [11], [12] and the other is System-Coupling Diagrams [13].

The Viable Systems Model (VSM) is presented in Fig. 5. It was developed by Stafford Beer in the 1970s [11] and is modeled after the manner in which a human 
being functions while interacting with its environment. A viable system has five interacting subsystems that may be mapped onto aspects of organizational structure. Systems 1-3 are concerned with the daily operations of an organization. System 4 is concerned with the strategical responses to the effects of external, environmental and future demands on the organization. System 5 is responsible for policy decisions within the organization as a whole to balance demands from different parts of the organization and steer the organization as a whole. System 5 is responsible to maintain the identity of the organization in order to promote a balance between stability and change. Any system of these 5 is also a viable system, which allows recursive decomposition of the model.

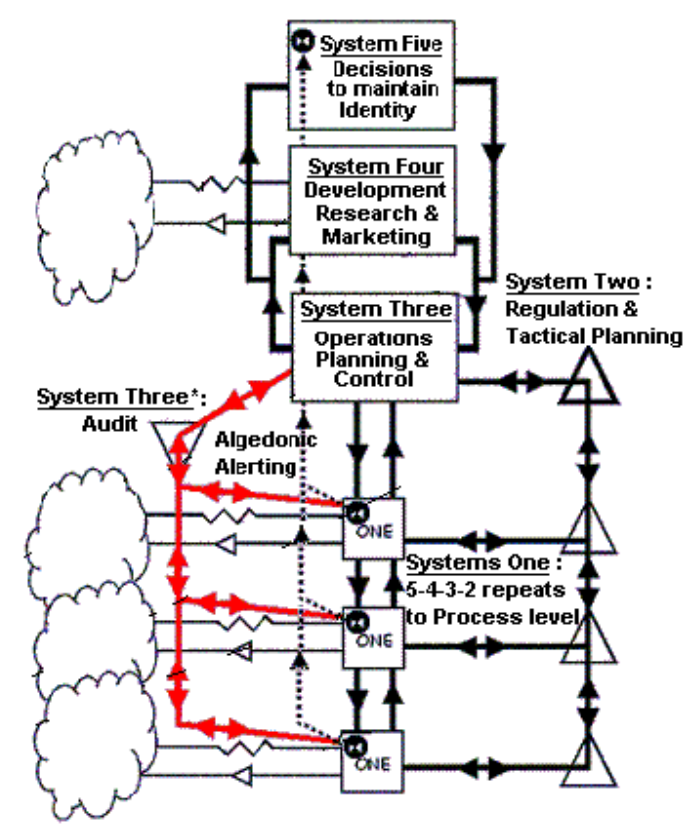

Fig. 5. Viable Systems Model from Wikipedia [12]

Besides systems 1-5, VSM includes Algedonic Alerts (from the Greek $\alpha \lambda \gamma o \varsigma$, pain and $\eta \delta \circ \varsigma$, pleasure) - e.g., alarms and rewards that escalate through the levels of recursion when actual performance fails or exceeds capability, typically after a timeout. It is the inclusion of the Algedonic Alerts within VSM which provides a strong relation to the three-layered enterprise model presented in this paper. There would exist different sets of BPTs and sensors for each system within the viable systems model resulting in BPI initiation when the sensors fire. It should also be realized that the BPTs within each system will actually relate to the operational, process improvement and strategic, dimensions as described in Section 4. While this is the case, based on the functions assigned to the different systems within VSM, some systems will have more of one class of BPTs than another. For example, system 5 , probably, will have more strategic BPTs than other systems. 
The idea of System-Coupling Diagrams comes from [13] and is represented in Fig. 6. It describes a general case when a particular situation in the system's environment causes a larger system, e.g., an enterprise, to create a respondent system, e.g., a project, to handle the situation. The respondent system is built from the assets the larger system already has. We used this idea in our work by interpreting business process instances as respondent systems. In addition to that, we have introduced sensors to discover situation that require building respondent systems. Execution rules, and start conditions of business process diagrams can be considered as control elements of respondent systems and sensors. The notation of Fig.1-4 was inspired by System-Coupling Diagrams.

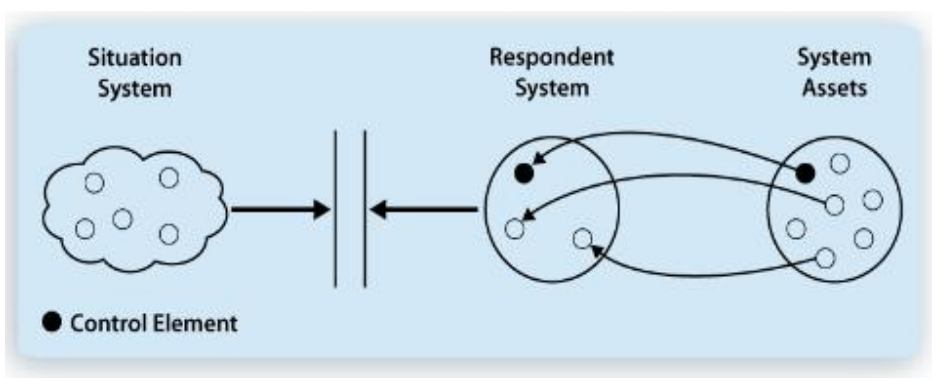

Fig. 6. System Coupling Diagrams from [13]

Business Process Management itself has a movement towards more flexible (agile) processes, which includes, but is not limited to the Adaptive Case Management, and flexible workflows. We do not overview the works related to these issues here, as they concern flexibility/agility in the frame of a particular process, not in the frame of the whole enterprise.

\section{Conclusion}

As follows from the title, our three-layered enterprise model is being built based on the reconciliation of Systems and Process Thinking. From Systems Thinking comes the view of the business process as a system, and the idea of cross-manning of business processes which creates a tighter cooperation between the parts of the enterprise system. From Process Thinking comes the need of having standardized ways of handling typical situations. Not having them for an organization means that any simple impulse from the outside needs to be processed in an ad hoc manner. An organization without standardized processes can be compared to a person that needs to think how to make each next step when walking along the street. Lack of standard processes is one of the weaknesses of small enterprises that make it difficult for them to compete with the big ones.

This paper begins with the saying "All models are wrong, ...", which is true for our three-layered model as for any other. However, we hope that this simplified model might actually reveal the level of agility of an enterprise and help in finding ways for its improvement. In other words, we believe the model might be useful for both analysis of the current situation in an enterprise, and for re-designing the enterprise. 
When considering business processes, more attention needs to be paid to having good sensors than having optimal processes. There is no need to have an optimal process for handling a customer order if we do not have a sensitive sensor that identifies who might need our products. In the same way, it does not make much sense to have a perfect product development process if we do not have a good sensor that can discover the needs for a new product before our competitors. The concept of crossmanning discussed in Section 7, seems to be one of the principles that could be useful in creating sensitive centers.

The model we suggested may seem to be totally event-driven, the system only reacts on external events. This may be so only if sensors are constructed in the way that they react to what becomes visible for every-one, e.g., economic crises of 2008. Having sensitive sensors makes the system proactive. The sensors can early on catch the trends that have not yet produced visible results. Applying Systems Thinking [6,7] can help in creating such sensors. Returning to the example of financial crises of 2008, having a sensor based on the H. Minsky financial instability hypothesis [14], could have helped to avoid the severity of the crises.

As far as process optimization is concerned, in the highly dynamic environment, this concept should be taken with caution. The more optimized a process is, the more difficult it will be to change it. The less optimal process that is easier to change can be much more "optimal" in the long run. In the practical plan, we need to move from the totally prescriptive definitions of the process execution rules to the constraint-based definitions - a combination of guidelines and restrictions, which allow and require creativity from the process participants handling the instance/case. In the scientific plan, we need to abandon the idea of a process as a flow of operation or events, and start considering it as a trajectory in a multidimensional state-space [15].

As far as computerized systems to support execution of business processes are concerned, the cross-manning concept requires moving the focus to facilitating collaboration/communication between the members of heterogeneous teams. Translating this requirement into the architecture of business processes support systems, there is a need to reconsider current fixation on the conveyor belt principle (workflow engines) in favor of the construction site metaphor of the shared spaces (such as different social software uses) as suggested in [16,17]. In addition, there is a need to have tools that allow the process participants themselves to design and/or change process definitions and adjust the support system to those changes. An example of such a tool is presented in [18].

For now, our three-layered model is just an idea of creating a new modeling technique, and we cannot present its empirical validation at this moment. Quite a lot of research and practical work is needed to convert it into practical modeling notation, and methodology. The promising thing here is that this can be done stepwise. As was shown in section 7 , the underlying thinking can readily be applied for understanding some situations. The first step in creating a methodology can be quite simple - design a technique that helps list all enterprise processes, classify them according to the scheme in Section 4, and describe what kind of a sensor each of them has. Based on this list one can start debating the presence, efficiency and sensitivity of existing sensors before raising the issue of optimization of the processes themselves. 
Acknowledgment. The first draft of the material presented in this paper was discussed in the LinkedIn group Systems Thinking World [10]. The authors are grateful to all members of the group who participated in the discussions, as their comments helped us to considerably improve the draft.

\section{References}

[1] Box, G.E.P.: Wikiquote, http://en.wikiquote.org/wiki/George_E._P._Box

[2] Weske, M.: Business Process Management. Concepts, Languages, Architecture. Springer, Heidelberg (2010)

[3] Harmon, P.: Business Process Change. A Guide for Business Managers and BPM and Six Sigma Professionals. Morgan Kaufmann Publishers, San Francisco (2007)

[4] Stelzer, D.: Enterprise Architecture Principles: Literature Review and Research Directions. In: Dan, A., Gittler, F., Toumani, F. (eds.) ICSOC/ServiceWave 2009. LNCS, vol. 6275, pp. 12-21. Springer, Heidelberg (2010)

[5] Proper, E., Lankhorst, M.M., Schönherr, M., Barjis, J., Overbeek, S. (eds.): TEAR 2010. LNBIP, vol. 70. Springer, Heidelberg (2010)

[6] Checkland, P.: Systems Thinking, Systems Practice. Wiley, Chichester (1999)

[7] Jackson, M.C.: Systems Thinking: Creative Holism for Managers. Wiley, Chichester (2003)

[8] Malone, T., Crowston, K., Lee, J., Pentland, B., Dellarocas, C., Wyner, G., Quimby, J., Osborn, C., Bernstein, A., Herman, G., Klein, M., O’Donnell, E.: Towards a Handbook of Organisational Processes. Management Science 45(3) (1999)

[9] Sherehiy, B., Karwowski, W., Layer, J.K.: A review of enterprise agility: Concepts, frameworks, and attributes. International Journal of Industrial Economics 37, 445-460 (2007)

[10] Systems Thinking World group, http: / / www. linkedin.com/ groups / Systems-Thinking-World-2639211

[11] Beer, S.: Brain of the Firm; Allen Lane. The Penguin Press, London (1972)

[12] Viable Systems Model, Wikipedia, http://en.wikipedia.org/wiki/Viable_System_Model

[13] Lawson, H.: A Journey Through the Systems Landscape. College Publications (2010)

[14] Minsky, H.P.: The Financial Instability Hypothesis. The Jerome Levy Economics Institute Working Paper No. 74. SSRN (May 1992),

http: / / ssrn. com/abstract=161024 doi:10.2139/ssrn.161024

[15] Khomyakov, M., Bider, I.: Achieving Workflow Flexibility through Taming the Chaos. In: Patel, D., Choudhury, I., Patel, S., de Cesare, S. (eds.) OOIS 2000 - 6th International Conference on Object Oriented Information Systems, pp. 85-92. Springer, Heidelberg (2000)

[16] Bider, I., Perjons, E., Johannesson, P.: A strategy for merging social software with business process support. In: Muehlen, M.z., Su, J. (eds.) J.1, H.4, D.2. Part IV. LNBIP, vol. 66, pp. 372-383. Springer, Heidelberg (2011)

[17] Bider, I., Johannesson, P., Schmidt, R.: Experiences of Using Different Communication Styles in Business Process Support Systems with the Shared Spaces Architecture. In: Mouratidis, H., Rolland, C. (eds.) CAiSE 2011. LNCS, vol. 6741, pp. 299-313. Springer, Heidelberg (2011)

[18] iPB Reference Manual (on-line documentation). IbisSoft (2009), http://docs.ibissoft.se/node/3 